\title{
XVI.
}

\section{Casuistische Mittheilungen.}

\author{
Aus dem Pathologischen Institut in Bonn \\ von. \\ Dr. H. Jacobsthal, \\ Assistenten am Institut. \\ (Hierzu Taf. X.)
}

\section{Primäres Fibromyxom des linken Vorhofes.}

Obwohl die primären Geschwälste des Herzens bekanntlich zu den selteneren Befunden zählen, so sind dieselben doch, besonders in den letzten Jahren, vielfach zum Gegenstande von Mittheilungen gemacht worden. Was speciell die Myxome betrifft, so hat kürzlich Guth (21) ${ }^{1}$ ) an der Hand eines Falles die Bisherigen Arbeiten hierüber kurz zusammengestellt. Dieser Casuistik, welche noch durch den Fall Brodowski's (1), den Jäger's (16), sowie durch zwei weitere Fälle Ribbert's $(19,20)$ zu ergänzen ist, möchte ich eine im hiesigen Institute gemachte Beobachtung hinzufügen, welche insofern von Interesse ist, als sie meines Wissens den ersten Befund dieser Art bei einem Kinde darstellt.

Es handelte sich um ein vierjähriges Mädchen, welches unter der klinischen Diagnose „Mitral-Insufficienz" zur Section eingeliefert worden war.

Dem Protokoll vom 18. Juli 1898 (Professor Jores) entnehme ich Folgendes:

Mässig genährte Leiche mit starken Oedemen an den unteren Extremitäten und im Gesicht; Bauch stark aufgetrieben. Panniculus adiposus sowie die blasse Musculatur gering entwickelt; Unterhautzellgewebe ödematös. Aus der Baucbhöble entleert sich eine reichliche Menge klarer, gelblicher Flüssigkeit mit einzelnen Fibrinflocken. Peritonaeum blank. Lungen retrahirt. Linke Lunge frei, in der linken Pleurahōhle etwa $100 \mathrm{ccm}$ klarer Flüssigkeit in der rechten etwas weniger; rechte Lunge gleichfalls frei. Der Herzbeutel

1) Die Zahlen beziehen sich auf das am Schlusse gegebene LiteraturVerzeichniss. 


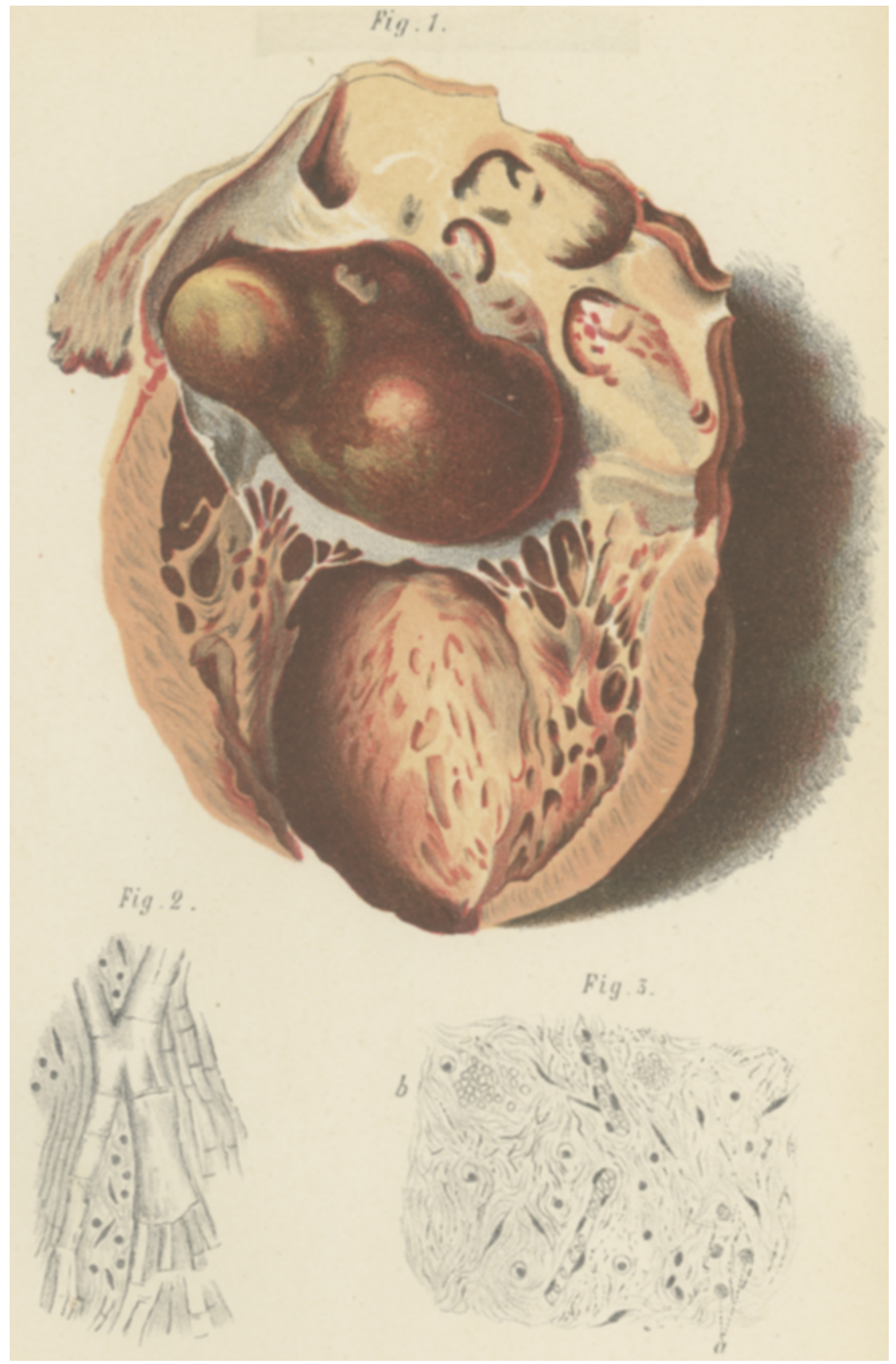


liegt in grosser Ausdebnung frei, enthält etwa $40 \mathrm{ccm}$ klarer Flüssigkeit; Pericard blank. Herz, besonders rechterseits, stark vergrössert, bis auf das Dreifache der Faust des Individuums. Aus dem rechten und linken Vorbof entleert sich nur wenig flüssiges Blnt. Recbter Vorhof weit, mit lockerem Cruor stark gefüllt, Trabekel treten siark hervor. Rechter Ventrikel von mittlerer Weite, enthält Cruor; Tricuspidalis intact. Conus arteriosus weit, Klappen der Pulmonalis nicht verändert. Musculatur kräftig entwickelt, namentlich in Conus arteriosus (7 $\mathrm{mm}$ dick). Linker Vorhof stark erweitert, mit lockerem Cruor gefüllt. Aus dem linken Herzohr ragt ein fast hühnereigrosser, graurotber, etwas gallertiger Tumor hervor. Wandung des Vorbofes dick; Mitralis intact, ebenso die Aortenklappen. Musculatur des linken Ventrikels kräftig, aber verhältnissmässig nicht so stark, wie rechts, obne Heerd-Erkrankungen.

Diagnose: Dedeme, Ascites, Hydrothorax, Myxom des linken Vorhofes, Hypertrophie des Herzens.

Ausserdem: Staung in den Lungen, der Leber und den übrigen Bauchorganen. Die genauere Betrachtung der Geschwulst ergiebt folgenden Befund:

Der etwa hübnereigrosse $(6: 3: 3 \mathrm{~cm})$ Tumor setzt sich aus einem grösseren und zwei kleineren Knollen zusammen, ist von glatter, spiegelnder Oberfläche und grösstentbeils dunkeirotber Farbe (kirschgelée-äbnlichem Ausseben), in den oberen Partien in der Nähe des Herzohres graugelblich, glasig durchscheinend, mit rothen Flecken. Seine Consistenz ist gallertig, schlotternd. Aus dem linken Herzohr gewissermaassen hervorquellend bängt er in das Atrioventricular-Ostium hinein, dieses ausfüllend. Die Neubildung sitzt dem Endocard des Herzohres mit einer Perhältnissmässig kleinen Basis fest auf; dieselbe ist unregelmässig rundlich umgrenzt, 1 bis $1 \frac{1}{2}$ crn im Durchmesser, und zeigt an mehreren Stellen ligamentähnliche Verbindungea mit dem Endocard, welche sich auf die Oberfläche des Tumors ausbreiten and allmahlich verlieren. Die Ansatzlinie der Geschwulst entspricht an der unteren, dem Herzen anliegenden Wand des Herzrohres, ziemlich genau der Herzobr-Vorhofsgrenze, nach oben hin rückt dieselbe allmäblich bis binter die Mitte der oberen Wandung des Herzobres. Von hier aus hat sich der Tumor nicht nur nach dem Vorhof, sondern auch nach der Herzohrspitze zu, diese ganz erfüllend, entwickelt.

Auf dem Durchschnitt zeigt derselbe nur an der Basis, wo er ohne scharfe Grenze in das Endocard übergebt, etwas dichteres, weisslich-graues Gefüge; von diesem Punkt aus strahlen auch weissliche, feine Gewebszüge divergirend in die gallertigen Massen, welche, wie die Oberfläche, grösstentheils dunkelroth erscheinen. Die oben beschriebenen gelblichen Stellen zeigen dieselbe Farbe auch in der Tiefe.

Wie scbon erwähnt, finden sich weder an der Mitralis, noch an dew Eadocard in der Umgebung der Gesebwulst Verdickungen oder sonstige Veränderungen. 
Die mikroskopische Untersuchung wurde sowobl am frischen Objecte, als nach Erbärtung in Alkohol vorgenommen (bei vorhergegangener Fixirung in Flem ing'seher, Zenker'seber Lösung, Kali bichrom.-Essigsäure-Gemisch nach Tellyesniezcky ${ }^{1}$ ), Formalin-Salzlösung (Jores). Die in Paraffu eingebctteten Stäeke wurden in Schnitte von 5-20 $\mu$ Dicke zerlegt und mit Hämalaun-Eosin, Saffranin, sowie nach v. Gieson gefärbt.

Die Grund-Substanz des Tumors (peripberische. Theile) erschien bei frischer Untersuchung ziemlich bomogen, amorph, auf Zusatz von Essigsäure feinkörnig, fädig. Bei Schnitten gehärteter Stücke finden sich in dieser (gleichfalls körnig-fädig erscheinenden) Grund-Substanz fibrilläre Elemente, und zwar ist das Verbälıniss der beiden Bestandtheile ein derartiges, dass an der Basis der Geschwulst und von dort aus weiter in die centralen Partien desselben sich fortsetzend, der fibrilläre Antheil, in der Peripherie der amorphe überwiegt. Bei Behandlung mit Thionin ${ }^{2}$ ) färbt sich letzterer stellenweise röthlich.

Die lockeren, wellig verlaufenden Fibrillenbündel durchflechten sich, ein Maschenwerk bildend, das von jener ungeformten Substanz ausgefülit, nach der Peripherie zu immer weiter wird, während die Fibrillen immer zarter und spärlicber werden. An der Basis stehen dieselben in continuirlichem Zusammenhang mit dem Bindegewebe des Endocards, und zwar nicht nur mit der oberflächlichen (glatte Muskelfasern führenden) Lage, sondern auch mit der tiefen, welche obne scharfe Grenze in das intermusculäre Bindegewebe übergeht. Dieses ist unter der Geschwulstbasis vermehrt, wobei die Muskelfasern des Vorbofes auseinandergedrängt erscbeinen. Von dieser tiefen Schicht aus streben Bindegewebsbündel mit Gefässen, die parallel zur Oberfăche verlaufenden Gewebszüge der darübergelegenen stellenweise durchsetzend, in den Tumor hinein: auch von letzterem aus findet ein Bineinstrahlen von Fibrillen statt. Der Reichthum an Gefässen ist ein ausserordentlicher. Die von der tieferen Schicht des Endacards abstammenden, zum Theil dickwandigen und weiten Gefässe, welche in den Stiel eintreten und in der Axe der Geschwulst weitergehen, verästeln sich unter Abnahme des Calibers, mit den Fibrillenbündeln verlaufend, und bilden ein reiches Capillarnetz, welches sich bis an die Oberfläche des Tumors erstreckt.

In grosser Ausdebnung finden sich Hämorrhagien, von der Basis an den grössten Theil der Geschwulst durchsetzend, sowohl frische, als ältere, wobei alle Uebergänge von in ihrer Gestalt unveränderten rothen Blutkörperchen, welche die Gewebemaschen stellenweise ausfällen, bis zu körnigem, freien Pigment sich vorfinden. Ueberraschend gross ist der Gehalt der Geschwulst an elastischen Elementen, welche bei der Färbung nach der neuen Weigert'schen Methode sehr deutlich hervortreten. Blane Netze, an der Basis dichter und aus stärkeren Fasern bestehend, nach der Peripherie zu weiter und zarter werdend, durchziehen den ganzen Tumor; be-

1) Arch. f. mikr. Anat., 1898, Bd. 52, Heft 2.

2) Untersuchung in Wasser. 
sonders in den Gefässwänden finden sich zablreiche elastische Elemente. Auch in der Näbe der Oberfläche sind noch reichliche Netze vorhanden, allerdings sind die Fasern hier stellenweise äusserst zart, doch deutlich blau gefärbt und von anderen ungefärbten Bindegewebs-Fibrillen scharf differenzirt. Zu erwähnen ist, dass man hier bisweilen solche sieht, die nicbt gleichmässig blau, sondern gekörnt erscheinen. Im Allgemeinen haben die elastischen Fasern die Neigung, mehr geradlinig, gestreckt zu verlaufen; im Gegensatz zu den mehr unter welligen Formen auftretenden BindegewebsFibrillen haben sie etwas Steifes an sich. Dieser Unterschied tritt besonders in den basalen und centralen Partien hervor, wo ibr Verlauf deutlich incongruent zu dem der Bindegewebsfasern ist, weniger in der Peripherie, wo sie gleichfalls theilweise wellig erscheinen. An der Basis hängen die elastischen Netze zusammen mit denen des Endocards. An den Fasern des letzteren finden sich folgende Abweichungen: Während dieselben in der Nachbarschaft der Geschwulst in der oberfächlichen Schicht mehrere dich untereinander gelegene Züge, welche anastomosirend parallel der Oberfläche entlang ziehen, bilden, in der tieferen in Form eines Netzwerkes die Fibrillen umspinnen, sind die ersteren da, wo der Tumor herauswächst, auseinandergedrängt, und erscheineñ im Vergleich zu dem stärker entwickelten Bindegewebe in ibrer Masse vermindert. Gleichzeitig ist auch die Richtung ihres Verlaufes derartig geändert, dass eine grosse Zahl ron ihnen senkrecht, sich mit den übrigen kreuzend, zur Oberfläche ziebt.

Verbältnissmässig arm ist die Geschwulst an zelligen Elementen. Es finden sich spindelige und sternförmige, stellenweise anastomosirende Zellen mit länglichem oder rundlich erscheinendem Kern und zartem Protoplasma, welche entweder den Fibrillenbündeln folgen, diese bekleidend, bisweilen in einer Reibe hintereinandel gelagert, theilweise aber auch in den Gewebemaschen liegen. Ausserdem sind noch kleine runde Kerne mit schmalem Protoplasmahof, dem Typus der kleinen Lymphocyten entsprechend, und grössere, protoplasmareichere Formen mit rundem oder polymorphen Zellieib in der amorphen Substanz vorhanden.

Eine Endothel-Bekleidung auf der Oberfläche nacbzuweisen, ist mir nicht gelungen.

Nach dem Vorhergehenden kann es nicht zweifelhaft sein, dass es sich in dem vorliegenden Falle bei dem Fehlen sonstiger Geschwülste im Körper um eine primäre Neubildung im Herzen handelt, und zwar um ein Fibromyxom. An der Basis und im Centrum entspricht der Bau durchaus dem des lockeren Fibroms; wenn ich von Myxofibrom spreche, so geschieht es einmal wegen des makroskopisch gallertigen Ansehens, ferner wegen des Vorhandenseins von Schleim in der amorphen Grundsubstanz, nachgewiesen durch Thionin, welches als zuverlässiges Reagens dafür 
bekannt ist, schliesslich desshalb, weil der Gehalt an Fibrillen in der ganzen peripherischen Zone ein so geringer ist, wie wir ihn in den Geweben, die als embryonales Bindegewebe oder Schleimgewebe bezeichnet werden, zu finden gewohnt sind.

In Betreff der systematischen. Stellung der Myxome stehen sich bekanntlich immer noch zwei Ansichten gegenüber: die eine welche dieselben als Geschwülste sui generis betrachtet und welche in den letzten Jahren wieder von $0 \mathrm{rth}^{\mathrm{T}}$ ) vertreten wurde, der in ödematösen Bindegewebs-Geschwülsten kein Mucin nachweisen konnte; die andere, von Koester ${ }^{2}$ ) stammend, der den Uebergang von Bingewebe in Schleingewebe in Folge von Staung, durch welche das in den collagenen Fasern enthaltene Mucin zur Aufquellung gebracht wird, behauptet. Dieser letzteren Anschauung pflichtet in neuerer Zeit Lubarsch ${ }^{3}$ ) bei, auch Kickhefel ${ }^{4}$ ) insofern, als er das Schleimgewebe als einen Status mucosus bezeichnet, in den die verschiedensten Gewebe übergehen können.

Unser Tumor zeigt alle Uebergangs-Stadien von einem lockeren Bindegewebe bis zu einem solchen Gewebe, welches von dem der Nabelschnur, das man doch als einen Typus von Schleimgewebe seit jeher bezeichnet hat, vicht principiell verschieden ist. Daher kann ich die myxomatösen Stellen nur als eine Modification, bezw. als einen Jugendzustand der fibromatösen auffassen. Dass thatsächlich Stauungen in der Geschwulst vorhanden gewesen sein müssen, ist ohne Weiteres klar, wenn man die Knickung und Zerrung des verhältnissmässig dünnen Stieles durch das Gewicht der herabhängenden Neubildung und die Herzbewegungen in Betracht zieht; auch sprechen die ausgedehnten Hämorrhagien dafür. Dass dieser Stauung ein Einfluss auf die gallertige Aufquellung der Geschwulst zuzuschreiben ist, kann wohl nicht von der Hand gewiesen werden. Warum nicht in allen Fällen Fibrome durch Oedem schleimhaltig werden $\left(\mathrm{Orth}^{5}\right)$, vermag ich nicht zu sagen. Es kann auf dem Alter des collagenen Gewebes be-

1) Nachr. der Kgl. Ges. d. Wiss. zu Göttingon. Matb.-physik. Klasse: 1895.

9) Sitzungsber. d. niederrh. Ges. Bonn 1881 .

3) in Lubarsch u. Ostertag, Ergebnisse der allg: path. Morphol. u. Phys. 1895.

4) Dieses Arch. Bd. 129 (1892).

5) a. a. 0 .

Archiv f. pathol. Anat. Bd. 159. Hft. 2. 
ruhen (Köster ${ }^{1}$ ), Rumler ${ }^{2}$ ), vielleicht gehört aber auch eine gewisse Disposition des proliferirenden Gewebes dazu, aufquellbare Intercellular-Substanz in genügender Menge zu bilden, zumal da sich der gallertige Zustand auch bei sehr kleinen, gefässlosen Geschwülsten des Endocards ${ }^{3}$ ) findet, und auch sonst (z. B. im Alter) schleimige Degeneration an den Herzklappen beobachtet wird $\left.{ }^{4}\right)$.

In der Literatur finden sich $21^{5}$ ) Fälle von primärem Myxom des Herzens beschrieben. Von diesen möchte ich 3 als nicht sicher von der Besprechung ausschliessen, und zwar die von Curtis (3), Wiegandt (6) und Jägers (16). Curtis selbst erklärt die von ihm beschriebene Neubildung auf der Mitralis für eine entzündliche, und nicht für eine echte Geschwulst. Allerdings hat mich die Lectüre dieser Arbeit von jener Ansicht nicht überzeugen können, vielmehr verhält sich der Fall in vieler Beziehung ganz analog den sonst beobachteten. Der Befund queryestreifter Muskelfasern in dem als Myxosarcom bezeichneten Tumor Wiegandt's lässt mir seine Zugehörigkeit zu dieser Gruppe fraglich erscheinen. Jägers Fall ist ungenau beschrieben, auch erregt die Bemerkung, dass die Geschwulst einem in Erweichung begriffenen Thrombus ähnlich war, Bedenken.

Der Ausgangspunkt unserer Neubildung ist das Bindegewebe des Endocards. An diesem unterscheidet man bekanntlich ${ }^{6}$ ) zwei Schichten, eine oberflächlichere, dichtere, welche glatte Muskelzellen enthält, und eine tiefere mit Blut-, Lymphgefässen und Nerven, welche ohne Grenzlinie in das interstitielle Gewebe des Herzmuskels übergeht. Die Betheiligung der letzteren Schicht an der Geschwulstbildung möchte jch für die wichtigere halten, da von dieser her die Gefässe des Tumors stammen, und auch das Hineinwuchern des Bindegewebes in denselben mit theilweiser Durchsetzung der parallel dem Endocard verlaufenden Gewebszüge

i) a. a. 0 .

2) Ueber Myxome und Schleimgewebe. Diss. Bonn 1881 .

3) Vgl. Ribbert's Fälle $(18,20)$, der kleinste Tumor $1 \frac{1}{2}: 1 \mathrm{~mm}$ gross.

4) Siehe Ziegler, Lehrbuch.

5) Keine einbeitliche Deutung hat der Fall Tannenbeim's (Verb. der 66. Vers. D. Naturf. und Aerzte zu Wien, 1894) erfahren.

6) Ranvier, Techn. Lehrb. d. Histol. 
nachweisbar ist; doch findet auch in der oberflächlichen Schicht eine Wucherung statt. Vergleicht man die Angaben hierüber bei den andern Fällen, soweit sich solche finden, so bestehen nur geringe Differenzen. Als Ausgangspunkt wird bezeichnet: die oberste (subendotheliale) Schicht (Lorne [2], Salvioli [7], Ribbert), die mittlere Schicht bei wahrscheinlicher Betheiligung der oberen (Boström [8]), das sub-endo-(peri-)cardiale Gewebe (Czapek ]11, 12]).

Eine Endothel-Bekleidung auf der Oberfläche konnte bei einigen kleineren, papillär gebauten'Tumoren (Ribbert, G a th [21]) nachgewiesen werden, bei anderen gelang dies nicht. Wie auch in dem vorliegenden Falle ist der Gefässgehalt der Geschwülste meist ein bedeatender, wie aus der Bezeichnung der Farbe: "dunkelroth" - zu entnehmen, besonders hervorgehoben wird der Reichthum an Blutgefässen von Salvioli, Boström, Jürgens; gefässlos sind die Fälle von Ribbert.

Beachtung verdient der Befund von elastisohen Fasern, nachgewiesen durch die Weigert'sche Methode. Da sich dieselben in der ganzen Geschwulst bis in die Peripherie hinein verfolgen lassen und sich alle Uebergänge von ziemlich dicken Fasern bis zu allerfeinsten finden, Hand in Hand gehend mit der Ausbildung der übrigen fibrillären Bestandtheile, so kann es sich nur um eine Neubildung von solchen Elementen handeln. Obwohl das elastische Gewebe seit der Erfindung electiver FärbeMethoden sehr in den Vordergrund des Interesses getreten ist, so ist doch die Frage nach der Neubildung desselben noch keineswegs eine abgeschlossene. Was die Geschwülste überhaupt angeht, so erwähne ich den Befund von elastischen Fasern in Enchondromen (Virchow ${ }^{1}$ )), im Herzmyxom (Lorne), in einem Myxoma hyalinum (Kollmann ${ }^{2}$ )), in einem Fibrosarkom (Suda kewits $\left(\mathrm{ch}^{3}\right)$ ). Neuere Arbeiten, die auf der Orceïn-Methode basiren, haben kein einheitliches Resultat ergeben. Für eine Neubildung sprechen sich aus Soffiantini ${ }^{4}$ ) (in Molluscum),

1) Geschwülste.

2) Dieses Arch., Bd. 68 (1876).

3) Le tissu élast, sa texture et son développement (russisch), Kiew 1882, cit. nach Loisel (Journ. de l'anat, Bd. XXXIII [1897]).

4) Travaux du laborat. d'histol. du collège do France 1891-98. 
sowie Kromayer ${ }^{1}$ ) (in einem Papillom, in weichen Fibromen). $\mathrm{Zur}$ entgegengesetzten Ansicht kommen Riehl ${ }^{2}$ ) in Bezug auf Hibrome der Haut, Da Mesnil ${ }^{3}$ ) bei Fibroma molluscum und Warzen, Schulz ${ }^{4}$ ) bei Cutis pendula, Papillomen, Warzen. Es hat hiernach den Anschein, als ob die Fälle sich in dieser Hinsicht nicht gleich verhalten. Auch ist der Einfluss des Mutterbodens zu berücksichtigen (hier reich an elastischen Elementen). Demgegenüber muss ich für den vorliegenden Fall daran festhalten, die unzweifelhafte Neubildung elastischer Fasern zu betonen. Ueber dje genaueren histologischen Vorgänge hierbei gedenke ich an anderer Stelle bäber einzugehen.

Was den Sitz der Geschwulst im Herzen angeht, so ist höchst auffällig, dass es in mebr, als der Hälfte der Fälle der linke Vorhof ist (von 19 Fällen $11 \mathrm{Mal}^{5}$ ), $4 \mathrm{Mal}$ die Tricuspidalis $^{6}$ ), je 1 Mal die Pulmonalis (Ribbert), die Innenfläche des linken Ventrikels (Martinotti (10)), des rechten Ventrikels (Czapels), die Herzspitze (Czapek).

Die Grösse unterliegt erheblichen Schwankungen: von kleinen, $1 \frac{1}{2}: 1 \mathrm{~mm}$ messenden Knöpfchen an bis zu den Tumoren von über Taubenei-Grösse (der grösste $8 \mathrm{~cm}$ lang (Berthenson (14)). Die grossen gehören ausschliesslich dem linken Vorhof an.

Ein Einfluss des Alters auf die Bildung der Geschwalst kann nur insofern constatirt werden, als sämmtliche bisher beobachtete Fälle Erwachsene betreffen; bei diesen finden sich aber die verschiedensten Altersstufen vom 31. bis zum 83. Jahre angegeben. Unser Fall ist der erste bei einem Kinde.

Auch das Geschlecht ist ohne Einfluss: von 15 Angaben betreffen 8 weibliche, 7 männliche. Personen.

Ueber die Aethiologie der Neubildung ist nichts bekannt. Elne congenitale Störung (in Zusammenhang mit Vorgängen

12. Monatsh. pract. Derm., 1894. (19).

2) Ref. in d. Monatsh. f. pract. Derm., 1893.

3) Arch. f. Dermat. u. Syph., 1893. (25.)

4) Ueber das Verhalten d. elast. Fasern in d. normalen u. path. veränd. Haut. Diss., Bonn, 1893.

5) Brodowski, Lorne, Bamberger, Salvioli. Bostroem, Yirehow, Jürgens, Berthenson, Robin, Marchand; Autor.

6) Debove, Czapek, Ribbert (2 Fälle). 
beim Verschluss des For. ovale) wurde von Marchand erwogen, doch führt derselbe dagegen an, dass der Sitz der Geschwulst nicht in allen Fällen dieser Stelle entspräche. In der That scheint mir ausser dem Falle Marchand's nur noch der von Salvioli diese Ansicht zu stützen; häufiger dagegen ist der Sitz im Herzohr (4 Mal $\left.{ }^{1}\right)$ ). Auch für einen entzündlichen Ursprung findet sich keinen Anhalt. Leichte Verdickungen des Endocards finden sich in etwa ein Drittel der Fälle angegeben, bei der überwiegenden Mehrheit aber war dasselbe intact. Gegen den entzündlichen Ursprúng spricht sich auch Ribbert aus, der. die Entstehung der Geschwulst auf Entwicklungs-Störungen aus überschüssig gebildetem Zellen-Material annimmt.

Nicht ganz zu widerlegen ist meines Erachtens die Frage, ob es sich bei diesen Geschwülsten, wenigstens bei einem Theil derselben, nicht am organisirte Thromben handeln könne. Czapek, der diese Frage aufwarf, konnte schon 15 Fälle, darunter 4 eigene Beobachtungen, dieser Art zusammenstellen. Allerdings ist die Entscheidung nicht schwierig, wenn sich in dem Tumor noch Reste thrombotischer Massen vorfinden; ist dagegen die Organisation beendigt, die Neubildung rein bindegewebig und durch Oedem aufgequollen, so wird die Aehnlichkeit mit ächter Geschwulstbildung eine ausserordentlich grosse $\operatorname{sein}^{2}$ ). Gegen diese Auffassung lässt sich geltend machen, dass für die Mehrzahl dieser Geschwülste bei dem Intactsein des Endocards sich keine Ursache für eine Thrombose auffinden lässt. Was die Folgen des Tumors für das Herz und ferner das Individuum betrifft, so waren etwa in der Hälfte der Fälle keine vorhanden, die kleine Geschwulst stellte lediglich einen:an der Leiche gewonnenen Nebenbefund dar ${ }^{3}$ ). Einmal trat plötzlicher

1) Lorne, Virchow, Robin, Autor.

2) A uf zwei andere Entstehungsweisen ähnlicher Bildungen machte Bostroem (D. Arch. f. Klin. Med. Bd. 55 (1895)) aufmerksam, nebmlicb 1. durch Blutung in die Musculatur des rechten Vorhofes, 2. durch Thrombose eines Varix des Vorhofes., Dieselben kommen für den vorliegenden Fall nicht in Betracht, da der Tumor von keiner bindegewebigen Membran bekleidet war.

3) Es sind dies die Fälle von Debove, Salvioli, Czapek (2 Fälle), Ribbert ( 3 Fälle), Gutb. 
Tod obne vorheriges Kranksein ein, wahrscheinlich durch Einklemmung des im linken Vorhof stehenden Tumors in das MitralOstium (Bostroem).

Die grösseren Geschwülste im linken Vorhof wirkten als Kreislaufhinderniss, verursachten eine Hypertrophie des Herzens und führten, klinisch einen Mitralfehler vortäuschend, unter den Erscheinungen der Herz-Insufficienz (Oedem, Hydrops u.s.w.) zum Tode (4 Fälle: Lorne, Bamberger, Berthenson, Autor). Embolien in verschiedenen Organen (Gehirn, Nieren, Lungen, Milz, Darm) wurden in vier Fällen beobachtet (Virchow, Berthenson, Robin, Marchand), darunter zwei Fälle mit plötzlichem Tod anscheinend gesunder Personen (Robin, Marchand). Im Embolus wurden Geschwulstzellen von Marchand nachgewiesen; wirkliche Metastasenbildung ist nicht bekannt.

Um das Wesentliche der Beobachtung zusammenzufassen, so fand sich bei einem vierjährigen Mädchen ein hühnereigrosses, primäres Fibromyxom des linken Vorhofes, welches eine starke Hypertrophie des Herzens, besenders des rechten, verursacht und unter den Zeichen der Herz-Insufficienz zum Tode geführt hatte. Mikroskopisch ist der Gehalt an neugebildeten elastischen Fasern beachtenswerth.

Zum Schlusse ist es mir eine angenehme Pflicht, Herrn Professor Koester für die Anfertigung des Aquarells, sowie Herrn Professor Jores für die Ueberlassung des Falles meinen herzlichsten Dank auszusprechen.

\section{Erklärungen der Abbildangen auf Tafel X.}

Fig. 1: Myxofibrom des linken Vorhofes. Nat. Grösse.

Fig. 2: Längsschnitt durch den Papillar-Muskel: unveränderte und verkalkte Muskelfasern, interstitielles (Granulations-)Gewebe. Vergr. 50 fach.

Fig. 3: Schnitt aus einer centralgelegenen Partie des Tumors.

a) Blutpigment. b) extravasirte rothe Blutkörperchen.

\section{Literatur.}

1. Brodowski, Denkwürdigk. d. Ges. d. Warsch. Aerzte, 1867. Citirt nach Wiegandt.

2. Lorne, Bull. de la Soc. anat. de Paris, 1869. 
3. Curtis, Arch. de physiol., $1871 / 72$ (IV).

4. Bamberger, Ueber zwei seltene Herzaffectionen. Wien. med. Woch.,

1872 (2). Koester, dieses Arch., Bd 55 (1872).

5. Debove, Bull. de la soc. anat. de Paris, 1873.

6. Wiegandt, Zur Casuistik der primären Neubildungen im Herzen. St. Petersb. med. Woch., 1876 (19).

7. Salvioli, Rivist. elin. di Bologna, 1878 (10).

8. Bostroem, Sitzungsber. d. phys.-med. Soc. zu Erlangen, 1880.

9. Vircbow, Charité-Annalen, VI, 1881.

10. Martinotti, Contribuz, allo studio dei tumori del cuore. Gaz. delle cliniche, 1886.

11. und 12. Czapek, Zur pathol. Anat. d. prim. Herzgeschwülste. Prager med. Woch., 1891.

13. Jürgens, Zur Casuistik der primären Herzgeschwülste. Berl. klin. Wochenschr., 1891.

14. Berthenson, Zur Frage der Diagnose primärer Neoplasmen d. Herzens. Dieses Arch., 132 (1893). Pawlowsky, Berlin. klin. Woch., 1895.

15. Robin, Note sur un cas de myxome du coeur. Arch. de médec. expér. et d'anat. path., 1893.

16. Jägers, Beitrag zur Kenntniss der primären Herzgescbwülste. Diss. München, 1893.

17. Marcband, Zur Kenntniss der Embolie und Thrombose der GehirnArterien u. s. w. Berl. klin. Woch., 1894.

18, 19, 20. Ribbert, Ueber Rückbildung an Zellen und Geweben und über die Entstehung der Geschwülste. Bibl. medic., 1897. Ne um an n, Inaug.Diss., Zürich, 1894. Ref. Ribbert, Deutsche med. Wochen., 1894.

21. Guth, Ueber einen Fall von papillärem Myxom auf d. Valv. tricusp. cordis. Prag. med. Woch., 1898 (8).

Nur im Referat zugänglich waren mir die Arbeiten von Brodowski, Salvioli und Martinotti.

II. Verkalkung von Herzmuskelfasern bei einem Kinde. Die über Verkalkung am Herzen in ziemlich erheblicher Zahl vorliegenden Beobachtungen betreffen vor Allem solche des Pericards, des Endocards (besonders an den Klappen), von Entzündungsproducten und Geschwülsten; dagegen sind die Angaben über solche der Muskelfasern spärlich und zerstreut. Mehrfach citirt ist der Fall Heschl's ${ }^{1}$ ), welcher diesen Befund an der Leiche einer 30jährigen Frau erhob; sehr instructiv die Mittheilung $R_{0} t^{\prime}{ }^{2}{ }^{2}$ ): Bei einem 29jährigen Mann, der im An-

1) Oesterr. Ztschs. f. prakt. Heilkunde, 1860.

2) Corresp.-Bl. f. Schweizer Aerzte, 1884. 